\title{
Predictors of objective cough frequency in pulmonary sarcoidosis
}

\author{
Aish Sinha ${ }^{1}$, Kai K. Lee ${ }^{1}$, Gerrard F. Rafferty ${ }^{2}$, Nadia Yousaf ${ }^{3}$, lan D. Pavord ${ }^{4}$, \\ James Galloway ${ }^{5}$ and Surinder S. Birring ${ }^{1}$
}

Affiliations: 'Division of Asthma, Allergy and Lung Biology, King's College London, King's Health Partners, London, UK. ${ }^{2}$ Child Health, School of Medicine, King's College London, King's Health Partners, London, UK. ${ }^{3}$ Dept of Oncology, St George's University Hospital Foundation Trust, London, UK. ${ }^{4}$ Dept of Respiratory Medicine, Nuffield Dept of Medicine, University of Oxford, Oxford, UK. ${ }^{5}$ Division of Rheumatology, King's College London, King's Health Partners, London, UK.

Correspondence: Surinder S. Birring, Division of Asthma, Allergy and Lung Biology, Denmark Hill Campus, King's College London, Denmark Hill, London SE5 9RS, UK. E-mail: surinder.birringlanhs.net

ABSTRACT Cough is a common symptom of pulmonary sarcoidosis. This study aimed to quantify cough frequency, and investigate its relationship with cough reflex sensitivity, pulmonary function and health status.

32 patients with pulmonary sarcoidosis were compared with 40 healthy controls. Cough reflex sensitivity to capsaicin, objective 24-h cough counts, cough-specific health status, cough severity and cough triggers were measured. The predictors of cough frequency in sarcoidosis were determined in a multivariate analysis.

Objective cough frequency was significantly raised in patients with sarcoidosis compared with healthy controls $(\mathrm{p}<0.001)$ and patients with cough had an impaired health status. Patients with pulmonary sarcoidosis had a heightened cough reflex sensitivity compared with healthy controls $(\mathrm{p}<0.001)$. Only cough reflex sensitivity was significantly associated with objective cough frequency in multivariate analysis, explaining $42 \%$ of the variance $(\mathrm{p}<0.001)$. There was no association between cough frequency, lung function, number of organs involved, chest radiograph stage or serum angiotensin-converting enzyme levels.

Cough is a common and significant symptom in patients with sarcoidosis. Ambulatory objective cough monitoring provides novel insights into the determinants of cough in sarcoidosis, suggesting that cough reflex sensitivity may be more important than lung function and other measures of disease severity, and this should be investigated further.

@ERSpublications

Cough reflex hypersensitivity is an important determinant of objective cough frequency in sarcoidosis http://ow.ly/W4q9e

This article has supplementary material available from erj.ersjournals.com

Received: Aug 172015 | Accepted after revision: Dec 012015 | First published online: Feb 042016

Support statement: This research was supported by King's College Hospital NHS Foundation Trust.

Conflict of interest: Disclosures can be found alongside the online version of this article at erj.ersjournals.com

Copyright OERS 2016 


\section{Introduction}

Sarcoidosis is a multisystem inflammatory disorder. The lungs are the most commonly affected organ followed by the skin and eyes [1]. The cause of sarcoidosis is not known, but exposure to antigens such as infectious, organic and inorganic agents in genetically susceptible individuals has been proposed $[1,2]$. Sarcoidosis is a T-lymphocyte-mediated disorder that leads to the formation and maintenance of granulomas. Cytokines, such as tumour necrosis factor, are important in recruiting inflammatory cells [1]. The clinical course of patients is highly variable and ranges from patients who are asymptomatic to those who progress to irreversible organ damage. The severity of pulmonary sarcoidosis is usually assessed with lung function tests and radiological imaging.

Cough is a common symptom of sarcoidosis. The prevalence of cough has been estimated to be between 3 and 53\%: Japan 3\%, Finland 33\%, Saudi Arabia 40\% and Turkey 53\% [3,4]. A chronic cough can lead to significant physical and psychological morbidity, and this can be assessed with cough-specific quality-of-life questionnaires [5-7]. Adverse symptoms frequently associated with cough include syncope, urinary incontinence, chest pain, sleep disturbance, anxiety and depression [5]. Heightened cough reflex sensitivity has been proposed as an important mechanism in a number of pulmonary disorders, such as idiopathic chronic cough [8], idiopathic pulmonary fibrosis (IPF) [9, 10] and bronchiectasis [11]. The recent development of ambulatory cough monitoring devices has facilitated the objective measurement of cough frequency $[12,13]$. In a study reported by KEY et al. [14], patients with IPF coughed frequently and this was associated with severe impairment in health-related quality of life. There is, however, a paucity of studies that have investigated cough in sarcoidosis. The aim of this exploratory study was to investigate cough frequency objectively with 24 -h cough monitoring, self-reported cough triggers, cough reflex sensitivity and impact on health status in unselected patients with pulmonary sarcoidosis. We also investigated the clinical predictors of objective cough frequency.

\section{Methods}

Subjects

Consecutive patients with pulmonary sarcoidosis attending a secondary-care sarcoidosis outpatient clinic were recruited. All had confirmed diagnosis of pulmonary sarcoidosis at the time of recruitment. The diagnosis of sarcoidosis and organ involvement was established when typical histological and clinical features were present using the ACCESS definitions [15]. Exclusion criteria were upper respiratory tract infection within the past 4 weeks, current smokers or ex-smokers (over 10 pack-years smoking history), presence of other coexisting respiratory conditions (such as emphysema present on computed tomography (CT) scan), symptoms of gastro-oesophageal reflux disease and rhinitis, and angiotensin-converting enzyme (ACE) inhibitor medication. The presence of cough was not an inclusion criteria. The objective cough frequency and capsaicin cough reflex sensitivity data from 40 age-range-matched healthy controls from a previous study were used for comparison [16]. All healthy subjects had normal spirometric values and were asymptomatic. The cough monitor and capsaicin challenge test methodology used in this study were similar to that used for healthy controls [16]. All subjects gave informed written consent and the study was approved by the local research ethics committee (East London and the City Research Ethics Committee 10/H0703/6).

\section{Questionnaires}

The Leicester Cough Questionnaire (LCQ) was used to assess the impact of cough on the patients' health status (health-related quality of life) and the cough-specific visual analogue scale (VAS, 0-100 mm) was used to assess cough severity. The LCQ is a validated chronic cough-specific health status questionnaire for adults [6]. It has 19 cough-specific questions that are divided into three domains (physical, psychological and social) and a seven-point Likert response scale. Scores for each domain range from 1 to 7 and the total score range is 3-21, with a higher score indicating a better health status. The presence of triggers and laryngeal symptoms associated with cough were recorded using a standardised novel questionnaire, the Cough Hypersensitivity Questionnaire [17] (CHQ) (see the online supplementary material). The CHQ comprises 23 items related to cough triggers, urge and laryngeal sensations associated with cough $[17,18]$. Patients respond on a categorical yes/no scale. The total score range is $1-23$, with a higher score indicating a greater number of triggers. The CHQ was developed in patients with chronic cough. A preliminary study reported higher CHQ scores in patients with refractory chronic cough (typically associated with heightened cough reflex sensitivity) compared with healthy subjects and subjects with cough associated with other chronic lung disease [17]. Self-reported sputum production was assessed with Item 2 from the LCQ [6].

\section{Physiological measures}

Lung function

Spirometry was measured in accordance with international guidelines [19]. 


\section{Cough frequency monitoring}

Cough frequency was recorded with the Leicester Cough Monitor (LCM). The LCM is a validated ambulatory cough monitor that consists of a portable MP3 sound recorder and free-field microphone, worn for $24 \mathrm{~h}$ in the patient's own environment [20,21]. The sound files were uploaded onto a computer for automated analysis using customised cough detection software described previously [12, 20]. Coughs were detected as single events whether occurring in isolation or bouts.

\section{Cough reflex challenge test}

Cough reflex sensitivity was assessed following cough monitoring with a capsaicin cough challenge test using a dosimeter method standardised to limit inspiratory flow [8,9]. Doubling concentrations of capsaicin $\left(0.49-1000 \mu \mathrm{mol} \cdot \mathrm{L}^{-1}\right)$ were administered until subjects coughed five or more times $\left(\mathrm{C}_{5}\right)$. Nebulised saline was randomly interspersed during the protocol to minimise the effect of anticipation. The concentration of capsaicin causing two and five coughs was calculated by interpolation.

\section{Protocol}

Patients attended hospital on two consecutive mornings between 09:00 and 12:00 $\mathrm{h}$. On the first day they completed the questionnaires and were set up for 24 -h cough monitoring. They returned the cough monitor the next day at the same time, and then underwent the spirometry and capsaicin cough challenge test. Serum ACE levels were obtained from clinical records if available within 3 months of recruitment to the study.

\section{Analysis}

Data was analysed using GraphPad Prism (version 5.00 for Windows; GraphPad, San Diego, CA, USA) and SPSS Statistics (version 17.0 for Windows; SPSS, Chicago, IL, USA). The distribution of data was assessed using the Kolmogorov-Smirnov test. Parametric data were expressed as mean \pm SD, whereas nonparametric data were expressed as median (interquartile range (IQR)). The cough reflex sensitivity and cough frequency and count data were log-transformed and presented as geometric mean \pm SD. Parametrically distributed data were analysed with independent sample t-tests to compare sample means, whereas comparison of nonparametric data was carried out using the Mann-Whitney U-test. All analyses included subjects with and without cough unless otherwise stated. For subgroup analyses, patients were grouped into those with normal and abnormal (raised) cough frequency (table 1 and online supplementary figure S2). The normal ranges for females and males have previously been reported: females $<5$ coughs $\cdot \mathrm{h}^{-1}$ and males $<2$ coughs $\cdot \mathrm{h}^{-1}[13,16]$. Correlations between variables were analysed with Pearson's correlation coefficient $(r)$ for parametric data and Spearman's correlation coefficient $(\rho)$ for nonparametric data. A p-value $<0.05$ was considered statistically significant. Predictors of objective cough frequency were assessed in patients with sarcoidosis using Pearson's and Spearman's correlations and general linear models. All subjects with sarcoidosis were included in the univariate and multivariate analyses $(n=32)$.

\section{TABLE 1 Impact of cough in sarcoidosis}

Sarcoidosis with cough

$\begin{array}{lc}\text { Subjects } & 17 \\ \text { Cough severity: VAS mm } & 53(20-66) \\ \text { Health-related quality of life: LCQ } & 14.8 \pm 3.7 \\ \quad \text { Total } & 4.8 \pm 1.3 \\ \text { Physical } & 5.0 \pm 1.3 \\ \text { Psychological } & 5.0 \pm 1.5 \\ \quad \text { Social } & \\ \text { Objective cough frequency } & 244 \pm 2 \\ 24 \mathrm{~h}^{-1 \#} & 10 \pm 2 \\ 1 \mathrm{~h}^{-1 \#} & \\ \text { Cough reflex sensitivity } & 1.9 \pm 1.9 \\ \mathrm{C}_{2} \mu \mathrm{mol} \cdot \mathrm{L}^{-1} & 6.8 \pm 3.2 \\ \mathrm{C}_{5} \mu \mathrm{mol} \cdot \mathrm{L}^{-1} & \end{array}$

Data presented as $\mathrm{n}$, median (interquartile range) or mean \pm SD. VAS: visual analogue scale; LCQ: Leicester Cough Questionnaire; $\mathrm{C}_{2}$ : concentration of capsaicin that caused two or more coughs; $\mathrm{C}_{5}$ : concentration of capsaicin that caused five or more coughs. The presence or absence of cough was assessed objectively using the Leicester Cough Monitor. The normal range of females and males is $<5$ and $<2$ coughs $\cdot \mathrm{h}^{-1}$, respectively $[13,20]$. This is a subgroup analysis of patients who have an objectively increased cough. Note: four patients did not self-report chronic cough. ${ }^{\#}$ : geometric mean \pm SD. 


\section{Results}

\section{Subject characteristics}

32 patients with sarcoidosis were recruited for this study. The characteristics of patients are shown in table 2. $63 \%$ of the patients were female and 66\% were of African-Caribbean ethnic origin. 29 patients had histological confirmation of diagnosis. Patients were symptomatic at presentation of their sarcoidosis. Two patients had a significant decline in lung function (change in forced vital capacity (FVC) $>10 \%$ or transfer factor of the lung for carbon monoxide (TLCO) $>15 \%$ ) within 3 months of recruitment. The indication for immunosuppressant therapy in those taking it at the time of study was pulmonary sarcoidosis with the exception of two patients (active cutaneous disease). Serum ACE levels were available for 23 patients, and the median (IQR) duration between the date of test and study recruitment was 3 (1-11) weeks. 18 patients self-reported a chronic cough with duration of $>8$ weeks at the time of study. The median (IQR) duration of cough for patients that reported chronic cough was 96 (28-144) weeks. No patient had a previous history of chronic cough that had resolved prior to entering the study. There were no significant differences in age, gender or smoking status between patients with sarcoidosis and healthy subjects. The median (IQR) duration of sarcoidosis since diagnosis was 24 (12-48) months. No subjects had diagnosed laryngeal or sinus sarcoidosis. 11 (34\%) patients were categorised as Scadding stage 4 based on the appearance of their chest radiograph.

\section{Cough frequency monitoring}

24-h cough counts were significantly higher in patients with sarcoidosis $(n=32)$ compared with healthy controls (geometric mean \pm SD: $67 \pm 5$ versus $18 \pm 3, \mathrm{p}<0.001$ ) (figure 1 ). Daytime (awake) cough counts were

\begin{tabular}{|c|c|c|c|}
\hline & Sarcoidosis patients & Healthy subjects & p-value \\
\hline Subjects n & 32 & 40 & \\
\hline Age years & $50 \pm 13$ & $49 \pm 13$ & 0.700 \\
\hline Female & $20(63)$ & $27(68)$ & 0.457 \\
\hline \multicolumn{4}{|l|}{ Ethnicity } \\
\hline Afro-Caribbean & $21(66)$ & & \\
\hline Caucasian & $8(25)$ & & \\
\hline South Asian $\#$ & $2(6)$ & & \\
\hline Other & $1(3)$ & & \\
\hline \multicolumn{4}{|l|}{ Smoking status } \\
\hline Current & $0(0)$ & $0(0)$ & \\
\hline Ex (<10 pack-years) & $7(22)$ & & \\
\hline Never & 25 (78) & $40(100)$ & \\
\hline \multicolumn{4}{|l|}{ Organs involved } \\
\hline Lungs & $32(100)$ & & \\
\hline Skin & $15(47)$ & & \\
\hline Eyes & $6(19)$ & & \\
\hline Nervous system & $4(13)$ & & \\
\hline Bone and joints & $4(13)$ & & \\
\hline Organs involved $n$ & $2 \pm 0$ & & \\
\hline FEV $1 \%$ pred & $72.2 \pm 19.1$ & $97.0 \pm 28.2$ & $<0.001$ \\
\hline FVC \% pred & $83.7 \pm 16.8$ & $100.9 \pm 29.0$ & $<0.001$ \\
\hline TLco \% pred & $66.5 \pm 11.5$ & & \\
\hline \multicolumn{4}{|l|}{ Scadding stage } \\
\hline $0 / 1$ & 8 & & \\
\hline II & 9 & & \\
\hline III & 4 & & \\
\hline IV & 11 & & \\
\hline \multicolumn{4}{|l|}{ Immunosuppressant ${ }^{\Upsilon}$} \\
\hline None & $19(56)$ & & \\
\hline Prednisolone & $10(29)$ & & \\
\hline Hydroxychloroquine & $3(9)$ & & \\
\hline Inhaled steroids & $2(6)$ & & \\
\hline Serum ACE level ${ }^{+}$IU.L $\mathrm{L}^{-1}$ & $75 \pm 37$ & & \\
\hline
\end{tabular}

Data presented as $\mathrm{n}(\%)$ or mean $\pm \mathrm{SD}$, unless otherwise stated. FEV1: forced expiratory volume in $1 \mathrm{~s}$; FVC: forced vital capacity; TLCO: transfer factor of the lung for carbon monoxide; ACE: angiotensin-converting enzyme. " : patients originating from India, Pakistan or Bangladesh; ๆ: immunosuppressant medications were those prescribed at the time of the study; ${ }^{+}$: normal serum angiotensin-converting enzyme (ACE) level $52 \mathrm{IU} \cdot \mathrm{L}^{-1}$. 


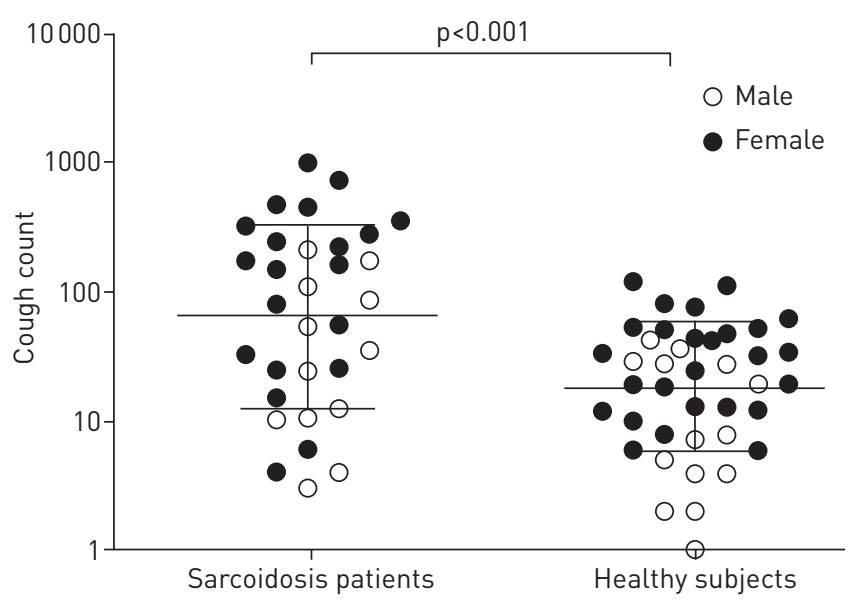

FIGURE 1 Comparison of $24-h$ cough counts between pulmonary sarcoidosis $(n=32)$ and healthy subjects $(n=40)$. Data are presented as geometric mean \pm SD. Objective cough counts per $24 \mathrm{~h}$ were measured using the Leicester Cough Monitor [12]. There was a statistically significant difference in 24-h cough counts between female and male subjects ( $p=0.024)$.

significantly greater than night-time (asleep) in patients with sarcoidosis $(n=32$; geometric mean \pm SD: $50 \pm 5$ versus $16 \pm 5$ coughs, $\mathrm{p}=0.008$ ). The cough characteristics of 17 patients with raised cough frequency are presented in table 1. Four of these subjects did not self-report a chronic cough. Female patients had significantly higher $24-\mathrm{h}$ cough counts compared with male patients $(\mathrm{n}=32$; geometric mean \pm SD: $110 \pm 5$ versus 29 $\pm 4, \mathrm{p}=0.024$ ) (figure 1 ). There was no significant relationship between cough counts and forced expiratory volume in $1 \mathrm{~s}\left(\mathrm{FEV}_{1}\right) \%$ predicted, FVC \% predicted, FEV1/FVC and TLCO (all $\mathrm{p}>0.05$ ). The correlation between cough counts and duration from diagnosis of sarcoidosis was $\mathrm{r}=0.05$, $\mathrm{p}=0.832$.

\section{Subjective assessment of cough}

Patients with pulmonary sarcoidosis and cough reported moderate cough severity (VAS) and significantly impaired health status (LCQ) $(n=17)$ (table 1). Cough impacted all health domains (table 1). The mean \pm SD self-reported sputum production score in patients who reported cough was $4 \pm 2$ (lowest possible score $=1$, highest possible=7). Patients reported a wide range of cough triggers; the commonest triggers were smoky atmosphere (54\% of patients), tickle sensation in the throat (49\%), perfume and scents (44\%), and irritation in the throat (43\%). $87 \%$ of patients reported one or more triggers, $69 \%$ reported two or more, $59 \%$ reported three or more and $13 \%$ reported none. The median (IQR) CHQ score was 6 (3-12). The median (IQR) CHQ score for patients with raised cough counts was 10 (5-14). Patients with raised cough counts reported more cough triggers than those with normal cough counts (median (IQR) CHQ score: 10 (5-14) versus 4 (2-9), $\mathrm{p}=0.008$ ). The internal reliability of CHQ (how well questionnaire items relate to one another and assess the same construct) was good (Cronbach's $\alpha=0.90)$.

\section{Cough reflex sensitivity}

Cough reflex sensitivity was heightened in patients with pulmonary sarcoidosis (all subjects $\mathrm{n}=32$ ) compared with healthy subjects (geometric mean $\pm \mathrm{SD} \mathrm{C}_{5}$ : $13.3 \pm 4.5$ versus $61.5 \pm 6.5 \mu \mathrm{mol} \cdot \mathrm{L}^{-1}, \mathrm{p}<0.001$ ) (figure 2). Female patients had increased cough reflex sensitivity compared with male patients (geometric mean \pm SD $C_{5}: 8.5 \pm 4.5$ versus $\left.28.6 \pm 3.4 \mu \mathrm{mol} \cdot \mathrm{L}^{-1}, \mathrm{p}=0.025\right)$. There was a significant correlation between $\mathrm{C}_{5}$ and serum ACE $(\mathrm{r}=0.72,95 \% \mathrm{CI} 0.43-0.87, \mathrm{p}=0.001)$ (online supplementary figure $\mathrm{S} 1$ ). There was no correlation between $24-\mathrm{h}$ cough counts and serum ACE $(\mathrm{r}=-0.23,95 \% \mathrm{CI}-0.59-0.20, \mathrm{p}=0.286)$. There was no significant correlation between $C_{5}$ and lung function $(\%)$ : FEV1 $(\rho=-0.09, p=0.641), F E V_{1} / F_{C}$ $(\rho=-0.14, p=0.452)$, FVC $(\rho=0.07, p=0.717)$ and TLCO $(r=0.29, p=0.176)$.

\section{Relationship between cough counts, health status, severity, triggers and reflex sensitivity}

There was a significant association between 24 -h cough counts and health status (LCQ total score: $\mathrm{r}=-0.61$, 95\% CI $-0.79--0.34, p<0.001$ ) (figure 3), cough severity (VAS: $\rho=0.62,95 \%$ CI $0.32-0.80, p<0.001$ ), cough triggers (CHQ total score: $\mathrm{r}=0.54,95 \%$ CI $0.23-0.75, \mathrm{p}=0.002$ ) (figure 4) and cough reflex sensitivity (log $\mathrm{C}_{5}: \mathrm{r}=-0.64,95 \% \mathrm{CI}-0.82--0.41, \mathrm{p}=0.000067$ (figure $5 \mathrm{a}$ ); $\log \mathrm{C}_{2}: \mathrm{r}=-0.62,95 \% \mathrm{CI}-0.79--0.34$, $\mathrm{p}=0.00017)$ in all patients $(\mathrm{n}=32) .24$ - $\mathrm{h}$ cough counts did not correlate with cough reflex sensitivity in healthy subjects $\left(\log \mathrm{C}_{5}: \mathrm{r}=-0.12,95 \% \mathrm{CI}-0.41-0.20, \mathrm{p}=0.472\right.$ ) (figure $5 \mathrm{~b}$ ). Patients with increased cough 


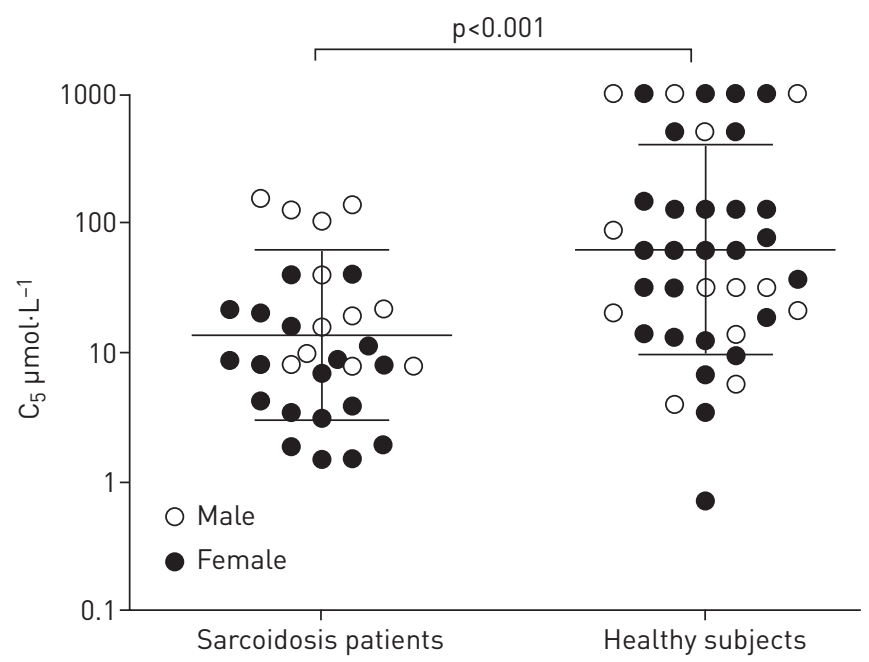

FIGURE 2 Comparing cough reflex sensitivity between patients with pulmonary sarcoidosis ( $\mathrm{n}=32$ ) and healthy subjects $(n=40)$. Data are presented as geometric mean \pm SD. $C_{5}$ : concentration of capsaicin that caused five or more coughs.

counts $(n=17)$ had a more heightened cough reflex compared with patients with normal range cough counts $(n=15)$ (geometric mean \pm SD $C_{5} 6.8 \pm 3.2$ versus $28.8 \pm 4.6 \mu \mathrm{mol} \cdot \mathrm{L}^{-1}, \mathrm{p}=0.005$ ) (online supplementary figure S2).

Cough reflex sensitivity $\left(\mathrm{C}_{5}\right)$ was significantly associated with the number of reported cough triggers (CHQ total score: $\mathrm{r}=-0.36,95 \% \mathrm{CI}-0.63--0.01, \mathrm{p}=0.045$ ) (online supplementary figure $\mathrm{S} 3$ ), and had a weak association with severity (VAS: $\rho=-0.32,95 \%$ CI $-0.61-0.06, p=0.090$ ) and health status (LCQ total score: $\mathrm{r}=0.32,95 \% \mathrm{CI}-0.04-0.60, \mathrm{p}=0.077)$ that approached statistical significance. The number of cough triggers (CHQ total score) was associated with cough severity (VAS: $\rho=0.57,95 \%$ CI $0.25-0.78, p=0.001$ ) (online supplementary figure S4) and health status (LCQ total score: $r=-0.68,95 \%$ CI $-0.83--0.43$, $\mathrm{p}<0.001$ ) (online supplementary figure S5), respectively.

\section{Predictors of objective cough frequency in sarcoidosis}

Table 3 summarises the relationship between patient characteristics and objective cough frequency in the univariate and multivariate models. In the univariate analyses, gender, reported sputum production and cough reflex sensitivity correlated significantly with cough frequency. There was a trend towards significance for age. There was no significant relationship between cough frequency and lung function (online supplementary figure S6), serum ACE, use of immunosuppressant medication, smoking status and chest radiograph Scadding stage. There was no significant difference between cough counts for patients with

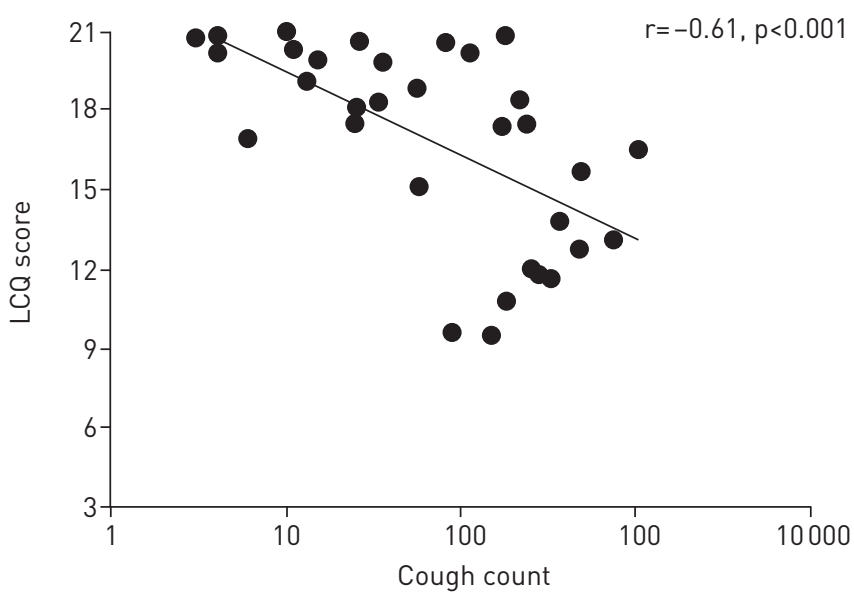

FIGURE 3 Relationship between 24-h cough counts and health-related quality of life ( $n=32$ ). Health-related quality of life was measuring using the Leicester Cough Questionnaire (LCQ) [6]. Objective cough counts per $24 \mathrm{~h}$ were measured using the Leicester Cough Monitor [12]. 


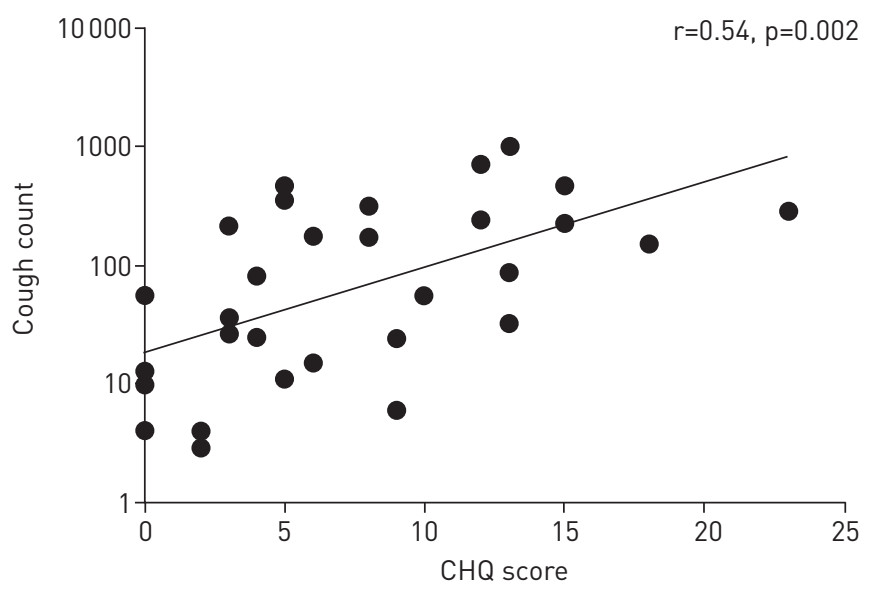

FIGURE 4 The association between self-reported cough triggers and 24-h cough counts ( $\mathrm{n}=32$ ). Objective cough counts per $24 \mathrm{~h}$ were measured using the Leicester Cough Monitor [12]. The Cough Hypersensitivity Questionnaire (CHQ) was used to assess cough triggers [17].

(Scadding stage 2-3) and without (Scadding stage 0-1) lung parenchymal disease $(\mathrm{p}=0.877)$. Significant influences on objective cough frequency were further explored in a multivariate model, including three of the most statistically significant variables in univariate analysis (gender, reported sputum production and $\mathrm{C}_{5}$ ). Incorporating gender, reported sputum production and cough reflex sensitivity $\left(\mathrm{C}_{5}\right)$ into the model explained $42 \%$ of the variance in cough frequency $(\mathrm{p}=0.001)$. Cough reflex sensitivity $\left(\mathrm{C}_{5}\right)$ remained a highly significant predictor of cough frequency within the multivariate model (table 3 ).

\section{Discussion}

This is the first study to investigate 24-h objective cough frequency and the impact of cough on health status in patients with pulmonary sarcoidosis. Cough frequency and reflex sensitivity were increased compared with healthy subjects, and were associated with a significantly impaired health status. Cough reflex hypersensitivity was the only independent predictor of objective cough frequency. There was no association between cough frequency and lung function or chest radiograph stage.

Cough frequency monitors are increasingly being used as assessment tools and are often the primary outcome measure in trials of antitussive therapy [21]. Cough frequency was significantly higher than healthy subjects, but less than that reported in patients with idiopathic chronic cough [12]. Approximately half of our patients with sarcoidosis coughed more frequently than healthy controls. As expected, a few patients with a raised cough frequency did not report a chronic cough. The discordance between objective and subjective measures of cough has been reported previously [20]. Patients with sarcoidosis had a diurnal variation in cough frequency, being significantly higher during the day compared with the night. This is consistent with findings in patients with chronic cough and healthy subjects [22]. Female patients with sarcoidosis had a higher cough frequency than males, consistent with studies of patients with an
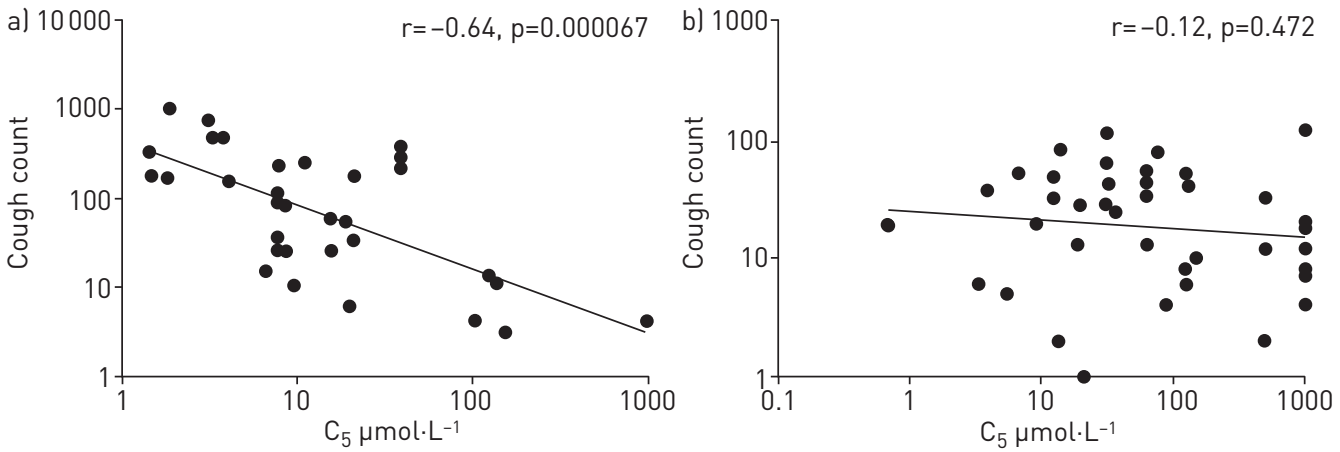

FIGURE 5 Relationship between cough reflex sensitivity and 24-h cough counts in a) patients with pulmonary sarcoidosis $(n=32)$ and b) healthy subjects $(n=40)$. Objective cough counts per $24 \mathrm{~h}$ were measured using the Leicester Cough Monitor [12]. C $_{5}$ : concentration of capsaicin that caused five or more coughs. Data for healthy subjects acquired from Yousaf et al. [16]. 


\begin{tabular}{|c|c|c|}
\hline Predictors of cough frequency & Correlation coefficient or variance $(95 \% \mathrm{Cl})$ & p-value \\
\hline \multicolumn{3}{|l|}{ Univariate analysis } \\
\hline Age & $0.32(-0.01-0.04)$ & 0.072 \\
\hline Gender & & $0.024^{+}$ \\
\hline $\mathrm{BMI}$ & $-0.15(-0.05-0.02)$ & 0.402 \\
\hline Race & & 0.190 \\
\hline \multicolumn{3}{|l|}{ Spirometry } \\
\hline FEV $1 \%$ pred & $-0.12(-0.02-0.001)$ & 0.510 \\
\hline FVC \% pred & $-0.12(-0.02-0.01)$ & 0.519 \\
\hline FEV $1 / F V C \%$ & $0.02(-2.03-2.21)$ & 0.934 \\
\hline TLCo \% pred & $-0.33(-0.65-0.10)$ & 0.127 \\
\hline Smoking status & & 0.982 \\
\hline Reported sputum production & $-0.36^{\text {ก }}(-0.64--0.00)$ & $0.044^{+}$ \\
\hline \multicolumn{3}{|l|}{ CRS to capsaicin } \\
\hline $\log C_{2}$ & $-0.62(-1.79--0.34)$ & $<0.001^{+}$ \\
\hline $\log C_{5}$ & $-0.64(-0.82--0.41)$ & $<0.001^{+}$ \\
\hline ACE levels & $-0.23(-0.59-0.20)$ & 0.286 \\
\hline Number of organs involved & $-0.11^{\pi}(-0.45-0.26)$ & 0.538 \\
\hline Use of immunosuppressant medications & & 0.153 \\
\hline Scadding chest radiograph stage & $0.26^{\text {ก }}(-0.11-0.56)$ & 0.155 \\
\hline \multicolumn{3}{|l|}{ Multivariate analysis } \\
\hline Model & $\mathrm{R}^{2}=42 \%$ & $<0.001^{+}$ \\
\hline Gender & & 0.454 \\
\hline Reported sputum production & -0.14 & 0.369 \\
\hline $\log C_{5}$ & -0.58 & $0.001^{+}$ \\
\hline
\end{tabular}

Data represents the ability of all the variables observed in this study in predicting cough frequency. Multivariate analysis was carried out using variables that were statistically significant in predicting cough frequency: gender, reported sputum production and $\log C_{5}$. Self-reported sputum production was assessed by Item 2 from the Leicester Cough Questionnaire [6]. Multivariate analysis was carried out using variables that were either statistically significant or approaching statistical significance in univariate analysis. BMI: body mass index; FEV1: forced expiratory volume in $1 \mathrm{~s} ; \%$ pred: \% predicted; FVC: forced vital capacity; TLCO: transfer factor of the lung for carbon monoxide; CRS: cough reflex sensitivity; $\mathrm{C}_{2}$ : concentration of capsaicin that caused two or more coughs; $\mathrm{C}_{5}$ : concentration of capsaicin that caused five or more coughs; ACE: angiotensin-converting enzyme. ${ }^{\#}$ : $n=32 ;{ }^{~ ๆ}$ : Spearman's correlation coefficient; ${ }^{+}$: statistically significant.

idiopathic chronic cough [16]. There was no significant relationship with smoking history, but there were no current smokers in our study and only seven ex-smokers. We did not find a relationship between cough frequency and the severity of pulmonary sarcoidosis assessed using traditional methods. There was no significant relationship between cough frequency and lung function, number of organs involved, use of immunosuppressive medications or chest radiograph stage. This suggests that cough may be independent of disease severity as assessed with clinical outcome measures. Patients with sarcoidosis who cough should undergo investigation for cough earlier to establish a diagnosis.

Cough reflex sensitivity was assessed by inducing cough with capsaicin. Capsaicin is a potent tussive stimulus and is the most widely used tussive challenge test. Cough reflex sensitivity was heightened in patients with pulmonary sarcoidosis compared with healthy subjects and was greater in patients with cough compared with those without cough. Female patients had a more sensitive reflex compared with males, consistent with known gender differences in other conditions [23-25]. CHOuDRY et al. [26] have also studied cough reflex sensitivity in patients with sarcoidosis. They found no difference in cough reflex sensitivity compared with healthy subjects. It is likely their study was underpowered to detect a difference as they only investigated eight patients, some had sarcoidosis and some had IPF. Furthermore, it is not possible to rule out a heightened cough reflex in the study by CHOUDRY et al. [26] since their presented data was combined with patients with IPF. Subsequent studies have reported heightened cough reflex sensitivity in IPF $[9,10]$. We found a significant association between cough reflex sensitivity and objective cough frequency in our patients with sarcoidosis. Cough reflex sensitivity was also associated with self-reported cough triggers and symptoms suggestive of laryngeal paraesthesia. A tickle sensation in the throat preceding cough is one of the most common manifestations of laryngeal paraesthesia and is thought to be a consequence of heightened cough reflex sensitivity [18]. Our data suggest that cough reflex sensitivity may be an important mechanism of cough in pulmonary sarcoidosis. Cough reflex sensitivity 
was, however, only weakly associated with self-reported cough severity. This is similar to findings in other chronic cough conditions, such as idiopathic chronic cough [20]. The weak relationship between subjective and objective measures of cough is well recognised; they potentially assess different domains of cough [27].

We did not investigate what influences cough reflex hypersensitivity in patients with sarcoidosis as this was beyond the scope of this study. The mechanism for the sensitisation of the cough reflex in idiopathic chronic cough is also poorly understood. Cough reflex hypersensitivity may be an important mechanism in many chronic respiratory disorders. For example, a weak-to-moderate relationship with cough frequency has been reported in patients with chronic obstructive pulmonary disease (COPD) [13] and idiopathic chronic cough [25]. Furthermore, a heightened cough reflex has been reported in IPF [25], acute cough [28], chronic cough [20, 24, 26, 29], bronchiectasis [11] and COPD [13, 30, 31]. The cough reflex consists of $\mathrm{C}$ fibre and $\mathrm{A} \delta$ nerve fibre endings in the airways that traverse through the vagus nerve to the brain stem and cortex. There is evidence of increased expression of neurotrophins, such as nerve growth factor and their receptors, on airway lymphocytes of patients with sarcoidosis [32]. Furthermore, DAGNell et al. $[33,34]$ have reported the presence of nerve growth factors and their receptors, such as TrkA, TrkB and TrkC, in sarcoid granulomas and alveolar macrophages. The neuronal mechanisms of cough should be explored further in patients with sarcoidosis. Cough in sarcoidosis may originate from a number of sites of the respiratory tract, such as the nose, sinuses, larynx, airways and lung parenchyma; this should also be investigated in further studies.

An unexpected finding of our study was the association between serum ACE levels and cough reflex sensitivity. Subjects with high ACE levels had reduced cough reflex sensitivity. This finding is surprising because patients with active disease tend to have higher serum ACE levels compared with those with inactive disease. ACE breaks down the potent airway tussive mediator bradykinin into inactive peptides [35]. This may be a potential explanation for the association that we found. We did not find an association between serum ACE and cough frequency. This may have been due to our small sample size. Our findings should be interpreted with caution because the relationship between cough reflex sensitivity and serum ACE levels was a post hoc analysis, and not the primary aim of our study. The association between cough, serum ACE and cough reflex sensitivity should be investigated in larger studies.

There was only a moderate relationship between subjective and objective measures of cough in pulmonary sarcoidosis. Objective cough frequency was moderately associated with cough severity VAS and health status. A weak-moderate association between subjective and objective measures of cough has also been described in patients with idiopathic chronic cough [27]. Subjective measures assess aspects of cough different to those measured with objective tools and are perhaps more important to patients since they represent their perception of illness. There are a number of cough assessment tools now available to assess sarcoidosis patients with cough. They are best used in combination to assess cough comprehensively [27]. It should, however, be noted that these tools were developed for patients with idiopathic chronic cough and have not formally been validated in sarcoidosis. It is not known if cough sounds from patients with sarcoidosis differ from other chronic respiratory disorders and whether this affects the accuracy of cough detection monitors. There have been some reports of differences in coughs sounds between respiratory conditions, but the importance of these findings is not known; furthermore, it is not possible to compare studies due to differences in recording equipment, microphones and sampling rates [36]. Cough frequency monitors have been used successfully in a number of respiratory disorders, such as COPD [13] and IPF [14], and the data from our study suggest that they may have a role in sarcoidosis. There is a need for validation studies of cough tools in patients with sarcoidosis.

There are some limitations to our study. We studied a small number of subjects and this may have led to clinically relevant imbalances in variables. Therefore, our findings need to be confirmed in larger studies, particularly those investigating the relationship between cough frequency and lung function. We did not assess patients for potential causes of cough, such as laryngeal/sinus disease; this should be investigated in future studies. Airway hyperresponsiveness may have been an important contributor to cough in some subjects; this should be evaluated in the future. It is possible we may have underestimated associations with cough reflex sensitivity with our methodology. We used a standardised method for the capsaicin challenge test as recommended by the European Respiratory Society [37]. A recent study has reported that the maximum number of coughs evoked by capsaicin $\left(\mathrm{E}_{\max }\right)$ may be able to discriminate patient groups better than current methods and therefore $\mathrm{E}_{\max }$ should be evaluated in future studies [38]. We did not study the immunopathology of sarcoidosis, and its relationship to cough, as this was beyond the scope of the study. We used data from a historical healthy control group [16] and did not record their ethnicity. The impact of ethnicity on cough is not known, but a recent worldwide survey of over 10000 patients with cough reported a largely uniform profile of patients [39]. It is possible that the follow-up criteria for our clinic (follow-up of incidental chest radiograph cases for only 2 years) could have led to a patient selection bias with a higher prevalence of cough. We performed a relatively large number of analyses for a small 
sample size and therefore some of our findings could be due to chance. Our data should be considered exploratory and be used to design larger studies.

We used a validated cough monitor, the LCM, that has been previously utilised in patients with idiopathic chronic cough, acute cough and COPD $[12,16]$. The accuracy of cough monitoring devices in sarcoidosis is, however, not known and therefore this could have impacted on the accuracy of our data; this should be assessed in the future. It is possible that cough may have been ameliorated in some patients taking immunosuppressive medication; hence, future studies should investigate incident cases before commencing treatment. We evaluated health status with the LCQ. We did not measure health status with sarcoidosis-specific measures, such as the King's Sarcoidosis Questionnaire [40] or the Sarcoidosis Assessment Tool [41]. They were not available at the time of the study. The LCQ is, however, a measure of both cough and related health status, and has been shown to be valid in a number of chronic respiratory conditions, such as chronic cough, COPD and IPF [22]. We assessed the radiological stage of sarcoidosis with chest radiographs. The limitations of radiograph staging are well recognised [42]. A CT scan-based radiological scoring system has recently been reported for sarcoidosis and shows promise, particularly for evaluating the extent of pulmonary fibrosis [43]. This and positron emission tomography scans, to assess disease activity, should be included in future studies of cough. A key question to address is whether cough in sarcoidosis relates to active disease or established pulmonary fibrosis. We assessed self-reported cough triggers, urge to cough sensations and laryngeal symptoms associated with cough using the CHQ [17]. This is not a validated questionnaire and only pilot data from idiopathic cough has been reported [17]. We used the CHQ to facilitate a structured assessment of cough triggers. Our preliminary data suggests the CHQ has good internal reliability (Cronbach's $\alpha=0.90$ ) and convergent validity (association with VAS, LCQ, cough frequency and cough reflex sensitivity) in sarcoidosis. The CHQ needs to be formally validated, but our data suggest it may be clinically useful.

The findings of our study suggest that cough is common in patients with sarcoidosis and is associated with significant impairment in health status. Our data also suggest that it is feasible to assess cough objectively with 24-h cough frequency monitors. Cough frequency was significantly raised in patients with sarcoidosis compared with healthy subjects. Cough reflex sensitivity was the only independent predictor of cough frequency. Cough reflex hypersensitivity deserves further study, as it may lead to a better understanding of the pathogenesis of cough in sarcoidosis.

\section{Acknowledgements}

We would like to thank the staff of the Lung Function Unit, King's College Hospital and all of the patients who took part in this study. Professor John Moxham (King's Health Partners, London, UK) provided valuable comments on the manuscript. We would also like to thank King's College Hospital NHS Foundation Trust for funding our research.

\section{References}

$1 \quad$ Iannuzzi MC, Rybicki BA, Teirstein AS. Sarcoidosis. N Engl J Med 2007; 357: 2153-2165.

2 Pietinalho A, Ohmichi M, Hirasawa M, et al. Familial sarcoidosis in Finland and Hokkaido, Japan - a comparative study. Respir Med 1999; 93: 408-412.

3 Pietinalho A, Ohmichi M, Hiraga Y, et al. The mode of presentation of sarcoidosis in Finland and Hokkaido, Japan. A comparative analysis of 571 Finnish and 686 Japanese patients. Sarcoidosis Vasc Diffuse Lung Dis 1996; 13: 159-166.

4 Harrison NK. Cough, sarcoidosis and idiopathic pulmonary fibrosis: raw nerves and bad vibrations. Cough 2013; 9: 9.

5 French CT, Irwin RS, Fletcher KE, et al. Evaluation of a cough-specific quality-of-life questionnaire. Chest 2002; 121: 1123-1131.

6 Birring SS, Prudon B, Carr AJ, et al. Development of a symptom specific health status measure for patients with chronic cough: Leicester Cough Questionnaire (LCQ). Thorax 2003; 58: 339-343.

7 Brignall K, Jayaraman B, Birring SS. Quality of life and psychosocial aspects of cough. Lung 2008; 186: Suppl. 1, S55-S58.

8 Prudon B, Birring SS, Vara DD, et al. Cough and glottic-stop reflex sensitivity in health and disease. Chest 2005; 127: $550-557$.

9 Doherty MJ, Mister R, Pearson MG, et al. Capsaicin induced cough in cryptogenic fibrosing alveolitis. Thorax 2000; 55: 1028-1032.

10 Hope-Gill BD, Hilldrup S, Davies C, et al. A study of the cough reflex in idiopathic pulmonary fibrosis. Am J Respir Crit Care Med 2003; 168: 995-1002.

11 Torrego A, Haque RA, Nguyen LT, et al. Capsaicin cough sensitivity in bronchiectasis. Thorax 2006; 61: 706-709.

12 Birring SS, Fleming T, Matos S, et al. The Leicester Cough Monitor: preliminary validation of an automated cough detection system in chronic cough. Eur Respir J 2008; 31: 1013-1018.

13 Sumner H, Woodcock A, Kolsum U, et al. Predictors of objective cough frequency in chronic obstructive pulmonary disease. Am J Respir Crit Care Med 2013; 187: 943-949.

14 Key A, Holt K, Hamilton A, et al. Objective cough frequency in idiopathic pulmonary fibrosis. Cough 2010; 6: 4.

15 Judson MA, Baughman RP, Teirstein AS, et al. Defining organ involvement in sarcoidosis: the ACCESS proposed instrument. ACCESS Research Group. A Case Control Etiologic Study of Sarcoidosis. Sarcoidosis Vasc Diffuse Lung Dis 1999; 16: 75-86.

16 Yousaf N, Monteiro W, Matos S, et al. Cough frequency in health and disease. Eur Respir J 2013; 41: 241-243. 
17 La-Crette J, Lee KK, Chamberlaine S, et al. The development of a cough hypersensitivity questionnaire (CHQ). Thorax 2012; 67: A127.

18 Vertigan AE, Gibson PG. Chronic refractory cough as a sensory neuropathy: evidence from a reinterpretation of cough triggers. J Voice 2011; 25: 596-601.

19 Miller MR, Hankinson J, Brusasco V, et al. Standardisation of spirometry. Eur Respir J 2005; 26: 319-333.

20 Birring SS, Matos S, Patel RB, et al. Cough frequency, cough sensitivity and health status in patients with chronic cough. Respir Med 2006; 1105-1109.

21 Spinou A, Birring SS. An update on measurement and monitoring of cough: what are the important study endpoints? J Thorac Dis 2014; 6: Suppl. 7, S728-S734.

22 Raj AA, Birring SS. Clinical assessment of chronic cough severity. Pulm Pharmacol Ther 2007; 20: 334-337.

23 Dicpinigaitis P, Rauf K. The influence of gender on cough reflex sensitivity. Chest 1998; 113: 1319-1321.

24 Kastelik J, Thompson R, Aziz I, et al. Sex-related differences in cough reflex sensitivity in patients with chronic cough. Am J Respir Crit Care Med 2002; 166: 961-964.

25 Kelsall A, Decalmer S, McGuinness $\mathrm{K}$, et al. Sex differences and predictors of objective cough frequency in chronic cough. Thorax 2009; 64: 393-398.

26 Choudry N, Fuller R. Sensitivity of the cough reflex in patients with chronic cough. Eur Respir J 1992; 5: 296-300.

27 Faruqi S, Thompson R, Wright C, et al. Quantifying chronic cough: objective versus subjective measurements. Respirology 2011; 16: 314-320.

28 O'Connell F, Thomas VE, Studham JM, et al. Capsaicin cough sensitivity increases during upper respiratory infection. Respir Med 1996; 90: 279-286.

29 O'Connell F, Thomas VE, Pride NB, et al. Capsaicin cough sensitivity decreases with successful treatment of chronic cough. Am J Respir Crit Care Med 1994; 150: 374-380.

30 Doherty MJ, Mister R, Pearson MG, et al. Capsaicin responsiveness and cough in asthma and chronic obstructive pulmonary disease. Thorax 2000; 55: 643-649.

31 Smith J, Woodcock A. Cough and its importance in COPD. Int J Chron Obstruct Pulmon Dis 2006; 1: 305-314

32 Ricci A, Mariotta S, Saltini C, et al. Neurotrophin system activation in bronchoalveolar lavage fluid immune cells in pulmonary sarcoidosis. Sarcoidosis Vasc Diffuse Lung Dis 2005; 22: 186-194.

33 Dagnell C, Grunewald J, Idali F, et al. Increased levels of nerve growth factor in the airways of patients with sarcoidosis. J Intern Med 2008; 264: 463-471.

34 Dagnell C, Grunewald J, Kramar M, et al. Neurotrophins and neurotrophin receptors in sarcoidosis; granulomas as a source of expression. Respir Res 2010; 11: 156.

35 Birring SS, Parker D, Brightling CE, et al. Induced sputum inflammatory mediator concentrations in chronic cough. Am J Respir Crit Care Med 2004; 169: 15-19.

36 Earis J, Smith JA. Analysis of the cough sound. In: Reid AE, ed. Acute and Chronic Cough (Lung Biology in Health and Disease Series). London, Taylor \& Francis, 2005; pp. 143-158.

37 Morice H, Fontana G, Belvisi M, et al. ERS guidelines on the assessment of cough. Eur Respir J 2007; 29: $1256-1276$.

38 Hilton E, Baverel $\mathrm{P}$, Woodcock A, et al. Pharmacodynamic modeling of cough responses to capsaicin inhalation calls into question the utility of the $C_{5}$ end point. J Allergy Clin Immunol 2013; 132: 847-855.

39 Morice A, Jakes A, Faruqi S, et al. A worldwide survey of chronic cough: a manifestation of enhanced somatosensory response. Eur Respir J 2014; 44: 1149-1155.

40 Patel A, Siegert R, Creamer D, et al. The development and validation of the King's Sarcoidosis Questionnaire for the assessment of health status. Thorax 2013; 68: 57-65.

41 Judson M, Mack M, Beaumont J, et al. Validation and important differences for the Sarcoidosis Assessment Tool: a new patient-reported outcome measure. Am J Respir Crit Care Med 2015; 191: 786-795.

42 Nunes $\mathrm{H}$, Uzunhan $\mathrm{Y}$, Gille $\mathrm{T}$, et al. Imaging of sarcoidosis of the airways and lung parenchyma and correlation with lung function. Eur Respir J 2012; 40: 750-765.

43 Walsh SL, Wells AU, Sverzellati N, et al. An integrated clinicoradiological staging system for pulmonary sarcoidosis: a case-cohort study. Lancet Respir Med 2014; 2: 123-130. 